\title{
GENERALIZATION OF THE EUCLIDEAN ALGORITHM FOR REAL NUMBERS TO ALL DIMENSIONS HIGHER THAN TWO
}

\author{
BY H. R. P. FERGUSON AND R. W. FORCADE
}

\begin{abstract}
A construction using integral matrices with determinant \pm 1 is given which has as corollaries generalizations of classical theorems of Dirichlet and Kronecker. This construction yields a geometrically convergent algorithm successfully generalizing the Euclidean algorithm to finite sets of real numbers. Applied to such a set this algorithm terminates if and only if the set is integrally linearly dependent and the algorithm gives absolute simultaneous integral approximations if and only if the set is integrally linearly independent. This development applies to complex numbers, can be used to give proofs of irreducibility of polynomials and yields effective lower bounds on heights of integral relations.
\end{abstract}

Let $\mathbf{Z}=$ rational integers, $\mathbf{R}=$ real numbers, $\mathbf{Z}^{n}=$ lattice points $\subset \mathbf{R}^{n}$ as row vectors, $\mathrm{GL}_{n}(\mathrm{Z}) \subset \mathrm{GL}_{n}(\mathrm{R})$ are $n$ by $n$ matrices with entries and invertible determinants in $\mathbf{Z} \subset \mathbf{R}$ resp. For $M=$ any matrix or vector, $M^{t}=$ transpose, $\operatorname{row}_{i} M=i$ th row, $\operatorname{col}_{j} M=j$ th column, height $(M)=\max$ absolute values of entries of $M$. The entries of $x \in \mathbf{R}^{n}$ are Z-linearly dependent iff there exists $0 \neq$ $m \in \mathbf{Z}^{n}$ such that $x m^{\boldsymbol{t}}=0, m=\mathbf{Z}$-relation for $x$. For $0 \neq x \in \mathbf{R}^{n}, x$ determines the line $x \mathbf{R}$ and orthogonal hyperplane $x^{\mathcal{1}}=\left\{y \in \mathbf{R}^{n}: x y^{t}=0\right\}$. A hyperplane matrix $Q$ with respect to $x$ is any matrix $x Q=0$ such that the columns of $Q$ transposed $\operatorname{span} x^{\perp}$. The hyperplane matrix is a key idea here in three aspects: (I) it permits estimates of heights of relations (Theorem 1), (II) it measures how closely the rows of a $\mathrm{GL}_{n}(\mathrm{Z})$ matrix are to the line $x \mathrm{R}$ (Lemma 1), (III) it underlies the definition of a crucial injection $\mathrm{GL}_{n}(\mathrm{Z}) \hookrightarrow \mathrm{GL}_{n+1}(\mathrm{Z})$ (Lemma 2). We exploit the nonuniqueness of $Q$.

THEOREM 1. Let $0 \neq x \in \mathbf{R}^{n}$. Then there exists a hyperplane matrix $Q$ such that height $m \geqslant 1 /$ height $A Q$ for $m$ any Z-relation for $x$ and any $A \in$ $\mathrm{GL}_{n}(\mathrm{Z})$.

SKetch OF PROOF. The parallelotope $|A|=\left\{\Sigma f_{j} \operatorname{col}_{j} A:\left|f_{j}\right| \leqslant 1 \leqslant j \leqslant n\right\}$ has easily characterized lattice points if $A \in \mathrm{GL}_{n}(\mathrm{Z})$. Let $I=$ identity matrix and define $Q$ to be the hyperplane matrix whose columns transposed are the vertices of the convex polytope $/ I / \cap x^{\perp}$.

$\mathrm{A} \mathrm{GL}_{n}(\mathrm{Z})$-algorithm is defined to be any construction (usually in response

Received by the editors March 26, 1979.

AMS (MOS) subject classifications (1970). Primary 10E45, 10F10, 10F20; Secondary 10F37, $12 \mathrm{A10}, 10 \mathrm{H} 05,02 \mathrm{E} 10$. 
to an $x$ ) of a sequence $\left\{M_{k}\right\}_{k \geqslant 1}, M_{k} \in \mathrm{GL}_{n}(\mathrm{Z})$. If $x M_{k}$ has a zero entry for some $k$ then the entries of $x$ are Z-linearly dependent: the algorithm terminates. If the height of $M_{k}^{-1} Q$ approaches zero as $k$ increases to infinity then the entries of $x$ are Z-linearly independent: the algorithm absolutely approximates $x$. A $\mathrm{GL}_{n}(\mathrm{Z})$-algorithm is split iff the algorithm terminates or absolutely approximates for every $x \in \mathbf{R}^{n}$.

LEMMA 1. If a $\mathrm{GL}_{n}(\mathrm{Z})$ algorithm is split and does not terminate for some $x$ then the distance of the rows of $M_{k}^{-1}$ to the line $x \mathbf{R}$ approaches zero as $k$ increases.

SKeTCh OF PROOF. For $x x^{t}=1$, set $Q=I-x^{t} x$ to get a line-hyperplane decomposition of any matrix $A=\left(A x^{t}\right) x+A Q$.

Define the $\mathrm{GL}_{2}(\mathrm{Z})$ algorithm $A_{2}$ by the following iteration. For $x=\left(x_{1}\right.$, $x_{2}$ ), let $x_{i}$ be of largest and $x_{j}$ of next largest absolute value. Replace $x_{i}$ by the $x_{i} \pm x_{j}$ of smaller absolute value. Then $A_{2}$ splits. We will give an uncountable collection of $\mathrm{GL}_{n}(\mathrm{Z})$ algorithms which split, $A_{n}(b), n \geqslant 2>1 / b>1$. For brevity we describe them by induction on $n$ by defining the injection $J: \mathrm{GL}_{n}(\mathrm{Z}) \subset$ $\mathrm{GL}_{n+1}(\mathrm{Z}), j$ is also an integer $1 \leqslant j \leqslant n+1$. Let $x_{(j)} \in \mathbf{R}^{n}$ be $x \in \mathbf{R}^{n+1}$ with the $j$ th entry deleted. For $A \in \mathrm{GL}_{n}(\mathrm{Z})$ set $T_{i}=\operatorname{row}_{i} A$ if $i<j$ or $T_{i}=\operatorname{row}_{i-1} A$ if $i>j, c_{i}=$ nearest integer to $T_{i} x_{(j)}^{t} x_{j} / x_{(j)} x_{(j)}^{t}$. Define $J(A) \in \mathrm{GL}_{n+1}(\mathrm{Z})$ to have a 1 in the $(j, j)$ position, zeros in that row, the $c$ 's in that column and $A$ the minor matrix of the 1 . Let $Q_{(j)}$ be $Q$ without the $j$ th row and $T_{i}=d_{i} x_{(j)}+$ $v_{i}$ orthogonally.

LEMmA 2. The injection map $J: \mathrm{GL}_{n}(\mathrm{Z}) \hookrightarrow \mathrm{GL}_{n+1}(\mathrm{Z})$ has the property that $\operatorname{row}_{i}(J(A) Q)=\operatorname{row}_{j} Q$ if $i=j$ and $\delta_{i} \operatorname{row}_{j} Q+n_{i} Q_{(j)}$ if $i \neq j$ where $\left|\delta_{i}\right|$ $\leqslant 1 / 2$.

To sketch the construction of $A_{n+1}(b)$ from $A_{n}(b)$ and the proof that $A_{n}$ splits, select the map $J$ by choosing $j$ to be the number of the row of $Q$ with least height. By induction $A_{n}$ splits; if $A_{n}$ does not terminate then for $0<\epsilon<$ $(b-1 / 2)$ height ( row $_{j} Q$ ) there exists a finite number of iterations of $A_{n}$ acting on $x_{(j)}$ yielding $x_{(j)} A^{-1}$ for a certain $A \in \mathrm{GL}_{n}(\mathrm{Z})$ with height $\left(n_{i}\right)<$ $\epsilon /$ height $Q$. By Lemma 2 , height $\left(\operatorname{row}_{i}(J(A) Q)\right)<b$ height $\left(\operatorname{row}_{j} Q\right)$ if $i \neq j$. Define one iteration of $A_{n+1}$ by $x \mapsto x(J(A))^{-1}$.

THEOREM 2. For every integer $n$ and real number $b, n \geqslant 2>1 / b>1$, the $\mathrm{GL}_{n}(\mathrm{Z})$ algorithm $A_{n}(b)$ splits, i.e., for every nonzero $x \in \mathbf{R}^{n}$ the sequence of matrices $M_{k}, k \geqslant 1$ is such that Termination) There exists a $k$ such that a column of $M_{k}$ is an integral relation among the entries of $x$ OR Absolute approximation) For every $\epsilon>0$ there exists an integer $K \geqslant 1$ such that for each $k \geqslant$ $K$ the rows of $M_{k}^{-1}$ give $n$ linearly independent lattice points in $\mathrm{Z}^{n}$, each within a distance $\epsilon$ of the line determined by $x$. 
THeOREM 3. Let $x=\left(x_{1}, x_{2}, \ldots, x_{n}\right) \in \mathbf{R}^{n}$ where $x_{1}=1, x_{2}, \ldots$, $x_{n}$ are Z-linearly independent real numbers. Then for every $\epsilon>0$ there exists an integral matrix $P \in \mathrm{GL}_{n}(\mathrm{Z})$ with first column $N, N^{t} \in \mathrm{Z}^{n}$ such that height $(N x-P)<\epsilon$.

THEOREM 4. A finitely generated spanning Z-module in $\mathbf{R}^{n}$ is dense in $\mathbf{R}^{n}$ if and only if every neighborhood of the origin contains a Z-basis for the $\mathbf{Z}$ module.

THEOREM 5. (a) The height of any quintic polynomial with integer coefficients having Euler's constant as a zero must exceed $10^{50}$.

(b) If $\zeta(3) / \pi^{3}$ satisfies an integral quadratic equation then at least two of the three coefficients exceed one hundred decimal digits in length.

(c) The first seven imaginary parts of the nontrivial zeros of the Riemann zeta function have no integral relations with heights $\leqslant 65$.

The proof of Theorems 3 and 4 involve applications of the construction represented in Theorem 2. The proof of Theorem 5 is given by exhibiting three integral matrices, from $\mathrm{GL}_{6}(Z), \mathrm{GL}_{3}(Z)$ and $\mathrm{GL}_{7}(Z)$ for parts (a), (b) and (c) respectively.

It is appropriate to give a very short historical commentary. The problem solved by the construction $A_{n}$ is rather old. $A_{2}$ is essentially in Euclid [Book X, Proposition 2]. The question of generalizing the Euclidean algorithm with an iterative process is implicit in Euler [Acta Acad. Sci. Imp. Petro. (1) 14 (1771), 188-214] and Lagrange [Leçons à l'école norm., 1795]. Hermite [Crelle's J. 40 (1850), 261-315] raised a related circle of questions. Iterative responses to Hermite's letters were made by Jacobi [Crelle's J. 69 (1868), 29-64] and Poincare [C. R. Acad. Sci. Paris 99 (1884), 1014-1016] and also a noniterative development by Minkowski [Acta Math. 26 (1902), 333-351], the latter subsequently improved by Mahler [J. Austral. Math. Soc. 4 (1964), 425-448]. Perron [Math. Ann. 64 (1907), 1-76] and Bernstein [Lecture Notes in Math. 207 (1971), 1161] followed the Jacobi line and Brun [Treizième Congr. Math. Scand. Helsinki (1957), 46-64] followed Poincaré with counterexamples and improvements.

Rosser [Proc. Nat. Acad. Sci. 27 (1941), 309-311] gave counterexamples to his proposal for dimensions above four. Simple counterexamples show that the Jacobi-Perron algorithm applied to an $n$-tuple can terminate without a relation. We have counterexamples to show that Brun's algorithm does not split for $n \geqslant$ 4. We have several examples that suggest Szekeres' [Ann. Univ. Sci. Budapest, Eötvös Sect. Math. 13 (1970), 113-140] algorithm does not split for $n \geqslant 5$. UTAH 84602

DEPARTMENT OF MATHEMATICS, BRIGHAM YOUNG UNIVERSITY, PROVO, 